\title{
The use of hyperbaric oxygen therapy in idiopathic sensorineural hearing loss: a case study
}

Chad Crigger ${ }^{1}$, Stephen Wilson MD, FACS ${ }^{1}$

\section{Author Affiliations:}

1. Joan C Edwards School of Medicine, Marshall University, Huntington, West Virginia

The authors have no financial disclosures to declare and no conflicts of interest to report.

Corresponding Author:

Chad Crigger

Marshall University Joan C. Edwards School of Medicine

Huntington, West Virginia

Email: crigger6@marshall.edu 


\section{Abstract}

Idiopathic sudden sensorineural hearing loss (ISSHL) is an otologic emergency that can provoke anxiety in the patient and can be clinically challenging for the practitioner. The natural history of the condition can be as varied as its possible etiologies. Adding to the clinical challenge is the current debate of treatment modalities available. In what follows, we provide a case detailing our treatment course, including the novel use of hyperbaric oxygen therapy (HBOT) as an emerging therapy for sensorineural hearing loss. Additionally, we provide a brief review of the current state of treatment options available in the armament for ISSHL. As no identifying information is contained in our discussion, we believe the privacy of the patient has been protected and, as such, feel our manuscript is exempt from prior Institutional Review Board (IRB) approval.

\section{Keywords}

Hyperbaric Oxygen Therapy, Idiopathic Sudden Sensorineural Hearing Loss

\section{Case Presentation}

A 49-year-old male with a history of a left-sided acoustic neuroma, stable in size since diagnosis, presented to the emergency department (ED) with a subjective sensation of sudden hearing loss lasting 46 hours in his right ear. The patient denied any recent trauma and stated he had tinnitus in the right ear. According to the patient, the hearing loss occurred quickly, beginning with tinnitus which led to a precipitous loss of all hearing within an hour. This occurred after undergoing a computed tomography (CT) angiogram for hypertension. Though the patient noted mild disequilibrium and right-sided fullness, he denied any vertigo or instability. After thorough evaluation, a $60 \mathrm{mg}$ glucocorticoid taper was initiated approximately 2 hours after arrival in the ED. The otolaryngologist then performed intratympanic (IT) glucocorticoid injection by injecting $0.4 \mathrm{cc}$ of $24 \mathrm{mg} / \mathrm{cc}$ of dexamethasone into the middle ear space. After initiating glucocorticoid therapy, the treating otolaryngologist recommended adjunctive hyperbaric oxygen therapy (HBOT) for treatment, at which point the patient was referred to our institution's wound center equipped with $\mathrm{HBO}$ chambers. In total, the patient received $20 \mathrm{HBO}$ treatments, each lasting 30 minutes and beginning at a pressure of 1 atmosphere absolute (ATA) before reaching a maximum of 2.4 ATA. At the conclusion of $20 \mathrm{HBO}$ sessions, the patient reported a considerable improvement in his hearing; a 30 decibels $(\mathrm{dB})$ improvement in hearing was confirmed by audiology.

\section{Introduction}

Sudden sensorineural hearing loss (SSHL) is defined clinically as a reduction in hearing greater than $30 \mathrm{Db}$, over at least three contiguous frequencies, in a period less than 72 hours. ${ }^{1}$ When clinical assessment is unable to determine a cause for hearing loss, this clinical picture is deemed idiopathic sudden sensorineural hearing loss (ISSHL). The natural history and clinical presentation of ISSHL can prove challenging for clinicians as the severity and timing of hearing loss varies by patient. For some patients the hearing loss is instantaneous and they are able to identify a specific time of onset. For others, the reduction in hearing is more gradual, occurring over hours to days. Though severity and temporal onset may vary, only one ear is usually impacted and prognosis depends on the severity of the initial hearing loss. 
Though it is difficult to measure the exact incidence of ISSHL, as patients who recover spontaneously may never actually seek treatment, estimates of incidence range from 5 to 20 per 100,000 people per year. While ISSHL may occur at any age, it most commonly occurs in patients ages 43 to $53 .^{2}$ In most cases of ISSHL the etiology is never determined, though viral cochleitis (i.e. Herpes simplex type I virus), microvascular events, and autoimmune disorders are suggested as likely causes. ${ }^{3}$ Though most of these conditions will not be identifiable on magnetic resonance imaging (MRI) with contrast, MRI does help rule out other causes of unilateral hearing loss, such as acoustic neuroma, perilymphatic fistula, Meniere's disease, vascular insufficiency, multiple sclerosis, or other conditions involving the central nervous system that may only be discovered on MRI scan. Clinically, ISSHL is distinguished from other causes of sensorineural loss as most other causes typically result in bilateral loss that is gradual.

Despite ISSHL having an elusive etiology, there are certain staples in management and therapy. Oral glucocorticoids have historically been the first-line treatment, administered promptly after diagnosis, ideally within two weeks. Treatment should begin at $1 \mathrm{mg} / \mathrm{kg} /$ day (to $60 \mathrm{mg}$ maximum) given as a single dose for 10-14 days. IT glucocorticoids have also been suggested for treatment, especially in patients who do not respond to oral glucocorticoids, however IT steroids have also been studied for initial treatment both with and without oral glucocorticoids. IT glucocorticoids may also be considered in patients who may not be able to tolerate oral options well, such as diabetics or those affected by side effects. For IT glucocorticoids, many dosing regimens have been offered, however dexamethasone dosed between 10 to $24 \mathrm{mg} / \mathrm{ml}$ is typical. $^{3}$

An emerging therapy for ISSHL is HBOT, which has shown promise in recent years. While science continues to elucidate the underlying mechanism of HBOT in ISSHL, its use in our patient and the benefit it provided lend evidence for continued research on the topic. In what follows, we seek to share our experience with HBOT for ISSHL in the case of our patient, and provide a brief review of the current state of HBOT and its role in treating ISSHL.

\section{Discussion}

Though the aforementioned definition of ISSHL provides clear diagnostic criteria, ISSHL still presents a clinical challenge for otologists, particularly when the etiology is unclear. While theories of potential causes abound, viral infection of the inner ear is believed to be the cause in the majority of cases. ${ }^{4,5}$ Even more perplexing than determining etiology in ISSHL is the fact that many patients, with some estimates as high as $65 \%$ of patients, completely recover functional hearing levels spontaneously, regardless of the type of medical treatment received. ${ }^{6}$

Glucocorticoids, whether systemic, intravenous, or injected into the intratympanic space, have long been considered the standard of therapy in ISSHL. Oral steroids remain the current standard first-line therapy, despite their unproven efficacy, given their generally acceptable adverse-event rates and the likelihood of providing the greatest recovery of hearing when initiated within the first 1 to 2 weeks after symptoms onset. ${ }^{2}$ When initiated, oral prednisone or methylprednisolone were associated with higher rates of improvement compared to placebo (61\% vs 32\%), however the rates of improvement observed in the corticosteroid-treated group mirrored the rate of recovery in patients not receiving any treatment $(65 \%)$, which aligns with the 
natural history of ISSHL. ${ }^{6,7}$ The underlying mechanism of corticosteroid therapy in improving ISSHL is that it works through reducing inner ear inflammation and edema. ${ }^{6}$

IT corticosteroids work on this same principle, but offer a higher perilymph drug concentration than systemic approaches, without the undesired metabolic and catabolic effects associated with systemic application. Additionally, IT corticosteroid therapy has often been referred to as "salvage therapy" in patients who did not respond to systemic therapy. Studies considering the use of IT corticosteroids as primary treatment are still underway, and current consensus supports their continued use as an adjunctive therapy. To add to the confusion in possible therapies, many other treatments, including stellate ganglion blockage, vasodilators, anticoagulant agents, plasma expanders, and diuretics have all been applied in ISSHL treatment, with varying degrees of success, as these medications are used to improved blood circulation and increase the oxygen pressure in the inner ear. ${ }^{2}$

However, our neurootologist's recommendation to begin HBOT sheds light on a newer treatment modality for ISSHL that is currently the subject of great research interest. ISSHL is one of the newest indications approved by the Undersea and Hyperbaric Medical Society's HBO Therapy Committee. In 2002 Aslan et al. demonstrated that the addition of HBOT to the conventional treatment for ISSHL (e.g. glucocorticoids) significantly improves outcomes, especially in patients younger than 50 years. $^{8}$ In their prospective randomized trial, Topuz et al. were amongst the first prospective studies to reaffirm the findings of Aslan et al., even to the sharp age demarcation of 50 years. ${ }^{9}$ Still others report HBOT improves hearing, but that the clinical significant of the level of improvement is unclear. ${ }^{10}$

Employing HBOT in the treatment of ISSHL is theorized to increase the partial pressure of oxygen and oxygen concentration in the inner ear to ultimately improve microcirculation. Under hyperbaric conditions, inhaled oxygen increases the oxygen tension of the perilymphatic fluid by $450 \%$ of its initial value, and this increased state remains for approximately 1 hour after termination of HBOT. The increase in arterial oxygenation diffuses from the capillary into the inner ear fluids, increasing the arterial partial oxygen saturation and influencing the oxygen tension of the inner ear, in such a way as to revive it. ${ }^{11}$ This is important as it has been reported that the oxygen tension of the perilymphatic fluid is decreased in patients with ISSHL.

ISSHL is a perplexing diagnosis, both for the patient and the practitioner. Even more intriguing are the variety of treatment modalities, despite efficacies that have yet to be proven consistently in the literature. The case of our patient highlights many important aspects of therapy in ISSHL: oral corticosteroids, followed by IT steroid therapy, in addition to adjuvant HBOT in a patient less than 50 years of age. Together, this multi-pronged approach helped our patient gain a significant recovery in his hearing. Even more importantly, our case provides further emphasis for continuing research on HBOT's promising role in treating ISSHL.

\section{Acknowledgements}

We would like to thank Dr. B Joseph Touma for his clinical expertise and help in treating our patient, who thankfully experienced a significant recovery of his hearing thanks to our teambased approach. 


\section{References}

1. Byl F. Sudden hearing loss: eight years' experience and suggested prognostic table. The Laryngoscope. 1984;94(5):647-661.

2. $\quad$ Rauch S. Idiopathic sudden sensorineural hearing loss. N Engl J Med. 2008;359(8):833-40.

3. Stachler R, Chandrasekhar S, Archer S et al. Clinical practice guideline: sudden hearing loss. Otolaryngol Head Neck Surg. 2012;146(3 Suppl):S1-S35.

4. Schuknecht H, Donovan E. The pathology of idiopathic sudden sensorineural hearing loss. Arch Otorhinolaryngol. 1986;243(1):1-15.

5. $\quad$ Linthicum F, Doherty J, Berliner K. Idiopathic sudden sensorineural hearing loss: vascular or viral? Otolaryngol Head Neck Surg. 2013;149(6):914-917.

6. Mattox D, Simmons F. Natural history of sudden sensorineural hearing loss. Ann Otol Rhinol Laryngol. 1977;86:463-80.

7. Wei B, Stathopoulos D, O'Leary S. Steroids for idiopathic sudden sensorineural hearing loss. Cochrane Database of Systematic Reviews. 2013. doi:10.1002/14651858.cd003998.pub3.

8. Wilson W, Byl F, N L. The efficacy of steroids in the treatment of idiopathic sudden hearing loss. A double-blind clinical study. Arch Otolaryngol. 1980;106(12):772-6.

9. Aslan I. Does the addition of hyperbaric oxygen therapy to the conventional treatment modalities influence the outcome of sudden deafness? Otolaryngol Head Neck Surg. 2002;126(2):121-126.

10. Topuz E, Yigit O, Cinar U, Seven H. Should hyperbaric oxygen be added to treatment in idiopathic sudden sensorineural hearing loss?. Eur Arch Otorhinolaryngol. 2004;261(7).

11. Bennett M, Kertesz T, Yeung P. Hyperbaric oxygen therapy for idiopathic sudden sensorineural hearing loss and tinnitus: a systematic review of randomized controlled trials. J Laryngol Otol. 2005;119(10). 